\title{
MAZUR'S INTERSECTION PROPERTY AND A KREIN-MILMAN TYPE THEOREM FOR ALMOST ALL CLOSED, CONVEX AND BOUNDED SUBSETS OF A BANACH SPACE
}

\author{
PANDO GRIGOROV GEORGIEV \\ (Communicated by William J. Davis)
}

\begin{abstract}
Let $\mathscr{V}$ (resp. $\mathscr{V}^{*}$ ) be the set of all closed, convex and bounded (resp. $w^{*}$-compact and convex) subsets of a Banach space $E$ (resp. of its dual $\left.E^{*}\right)$ furnished with the Hausdorff metric. It is shown that if there exists an equivalent norm $\|\cdot\|$ in $E$ with dual $\|\cdot\|^{*}$ such that $(E,\|\cdot\|)$ has Mazur's intersection property and $\left(E^{*},\|\cdot\|^{*}\right)$ has $w^{*}$-Mazur's intersection property, then

(1) there exists a dense $G_{\delta}$ subset $\mathscr{V}_{0}$ of $\mathscr{V}$ such that for every $X \in \mathscr{V}_{0}$ the strongly exposing functionals form a dense $G_{\delta}$ subset of $E^{*}$;

(2) there exists a dense $G_{\delta}$ subset $\mathscr{V}_{0}^{*}$ of $\mathscr{V}^{*}$ such that for every $X^{*} \in$ $\mathscr{V}_{0}^{*}$ the $w^{*}$-strongly exposing functionals form a dense $G_{\delta}$ subset of $E$. In particular every $X \in \mathscr{V}_{0}$ is the closed convex hull of its strongly exposed points and every $X^{*} \in \mathscr{V}_{0}^{*}$ is the $w^{*}$-closed convex hull of its $w^{*}$-strongly exposed points.
\end{abstract}

Let $\mathscr{V}$ be the set of all convex, closed, bounded and nonempty subsets of a real Banach space $(E,\|\cdot\|)$ and $\mathscr{V}^{*}$ be the set of all convex, $w^{*}$-compact and nonempty subsets of $\left(E^{*},\|\cdot\|^{*}\right)$ (the dual space of $E$ ). The Hausdorff metric between two subsets of $E$ is defined as follows:

$$
h(X, Y)=\inf \{\varepsilon>0: X \subset Y+\varepsilon B, Y \subset X+\varepsilon B\},
$$

where $X, Y \subset E$ and $B$ is the closed unit ball in $E: B=\{x \in E:\|x\| \leq 1\}$.

If $X$ and $Y$ in the above definition belong to $E^{*}$ and $B$ is replaced by $B^{*}$ (the closed unit ball in $E^{*}$ ), then the above formula defines the Hausdorff metric on $\mathscr{V}^{*}$.

It is well known that $(\mathscr{V}, h)$ is a complete metric space (see $[10$, p. 417]).

The set $S(X, l, \alpha)=\left\{x \in X:\langle x, l\rangle \geq \sup _{z \in X}\langle z, l\rangle-\alpha\right)$ is said to be a slice, depending on a subset $X \subset E$, on a continuous linear functional $l \in E^{*}$ and on $\alpha>0$.

The point $x \in X \subset E$ (resp. $x \in X \subset E^{*}$ ) is said to be a denting point (resp. $w^{*}$-denting point) if for every $\varepsilon>0$ there exist $l \in E^{*}$ (resp. $l \in E$ ) and $\alpha>0$ such that $x \in S(X, l, \alpha) \subset B(x ; \varepsilon)$. If in this definition $l$ does not depend on $\varepsilon$, then the point $x$ is said to be a strongly exposed (resp. $w^{*}$-strongly exposed) point and the functional $l$ is said to be a strongly exposing functional (resp. $w^{*}$-strongly exposing functional).

Received by the editors August 16, 1986 and, in revised form, July 24, 1987.

1980 Mathematics Subject Classification (1985 Revision). Primary 46B20.

Key words and phrases. Strongly exposed points, strongly exposing functionals, closed convex hull, Mazur's intersection property, sublinear functionals, Fréchet differentiability. 
There are many characterizations of the Banach spaces having the property that every $X \in \mathscr{V}$ (resp. every $X^{*} \in \mathscr{V}^{*}$ ) is the closed (resp. $w^{*}$-closed) convex hull of its strongly (resp. $w^{*}$-strongly) exposed points. One can obtain an information about this topic from the books: $[\mathbf{2}, \mathbf{3}, \mathbf{4}, \mathbf{6}]$. In the present paper the question is investigated, when this property is valid for almost all elements of $\mathscr{V}$ and $\mathscr{V}^{*}$ (in the Baire sense). It is true for $\mathscr{V}$ when $E^{*}$ is separable-this follows from [5], where it is proved also that every $l \in E^{*}, l \neq 0$ is a strongly exposing functional for almost all elements of $\mathscr{V}$.

The space $E$ (resp. $E^{*}$ ) has Mazur's (resp. $w^{*}$-Mazur's) intersection property if every $X \in \mathscr{V}$ (resp. every $X^{*} \in \mathscr{V}^{*}$ ) can be represented as an intersection of the closed balls which contain it [7 and 6, pp. 219, 230]. It was Mazur [11] who began the investigation of normed linear spaces possessing the above property. Further results on this topic were obtained by Phelps [12] and Sullivan [13]. Later Giles, Gregory and Sims [7, 6] gave many characterizations of the Banach spaces having Mazur's (resp. $w^{*}$-Mazur's) intersection property. One of them states: a Banach space $E$ has Mazur's (resp. $w^{*}$-Mazur's) intersection property if and only if the set of $w^{*}$-denting points for $B^{*}$ is dense in $S^{*}$ (resp. the set of denting points for $B$ is dense in $S$ ), where $B, B^{*}, S, S^{*}$ are respectively the closed unit balls and spheres in $E$ and $E^{*}$. Below we will see how this property is connected with a Krein-Milman type theorem for almost all elements of $\mathscr{V}$ and $\mathscr{V}^{*}$.

It is easy to see that the topology of $\mathscr{V}\left(\right.$ resp. $\left.\mathscr{V}^{*}\right)$ does not depend on the choice of the concrete equivalent norm in $E$ (resp. equivalent dual norm in $E^{*}$ ).

Further we need the following well-known lemma (see [2, p. 44]), whose proof is straightforward and is omitted.

LEMMA 1. For every slice $S\left(X, l_{0}, \alpha\right)$ there exists $\varepsilon>0$ such that $S(X, l, \alpha / 2) \subset$ $S\left(X, l_{0}, \alpha\right)$ for every $l \in E^{*},\left\|l-l_{0}\right\|^{*}<\varepsilon$.

LEMMA 2. Let $(M, d)$ be a metric space which contains at least two different points, $L$ be a dense subset of $M$ and $\nu$ be an integer, $\nu>1 / \operatorname{diam} M$. Then for every $n=\nu, \nu+1, \ldots$, there exists $L_{n} \subset L$ such that $d(x, y) \geq 1 / n$ for every $x$, $y \in L_{n}, x \neq y$ and $\bigcup_{n=\nu}^{\infty} L_{n}$ is a dense subset of $M$.

ProOF. Let $G_{n}$ be a subset of $L$ such that for every $x, y \in G_{n} x \neq y$ we have $d(x, y) \geq 1 / n$. Such $G_{n}$ exist for every integer $n \geq \nu$, for example $G_{n}=\left\{z_{1}, z_{2}\right\}$, where $z_{1}, z_{2} \in L$ and $d\left(z_{1}, z_{2}\right) \geq 1 / \nu$. Let $F_{n}$ be the set of all such $G_{n}$. By the Zorn lemma it follows that $F_{n}$ has a maximal element $H_{n}$ with respect to the usual order " $\subset$ ". We will prove, that $\bigcup_{n=\nu}^{\infty} H_{n}$ is a dense subset of $M$. Assume the contrary. Then there exists an open subset $U$ of $M$ for which $U \cap\left(\bigcup_{n=\nu}^{\infty} H_{n}\right)=\varnothing$. Take $x_{0} \in U \cap L$ and an integer $n_{0} \geq \nu$ such that $B\left(x_{0} ; 1 / n_{0}\right) \subset U$. Then $\widetilde{H}_{n_{0}}:=$ $H_{n_{0}} \cup\left\{x_{0}\right\}$ will belong to $F_{n_{0}}$ and $H_{n_{0}} \varsubsetneqq \widetilde{H}_{n_{0}}$, which is a contradiction with the maximality of $H_{n_{0}}$.

Denote by $N$ the set of all positive integer numbers.

LEMMA 3. Let $X \subset E$ be bounded. Then the strongly exposing functionals for $X$ form $a G_{\delta}$ subset of $E^{*}$ (perhaps empty).

ProOF. We will show, that the set $Y_{k}=\left\{l \in E^{*}: \inf _{\alpha>0} \operatorname{diam} S(X, l, \alpha)<1 / k\right\}$ is open for every $k \in N$. Let $k \in N$ be fixed and $l_{0} \in Y_{k}$. Then there exists $\alpha_{0}>0$ such that diam $S\left(X, l_{0}, \alpha_{0}\right)<1 / k$. By Lemma 1 it follows, that there exists $\varepsilon>0$ 
for which the conditions $l \in E^{*},\left\|l-l_{0}\right\|^{*}<\varepsilon$ imply $S\left(X, l, \alpha_{0} / 2\right) \subset S\left(X, l_{0}, \alpha_{0}\right)$. Hence $\inf _{\alpha>0} \operatorname{diam} S(X, l, \alpha)<1 / k$ which means that $Y_{k}$ is open. Obviously

$$
\bigcap_{k=1}^{\infty} Y_{k}=\left\{l \in E^{*}: l \text { is strongly exposing functional for } X\right\}
$$

and the proof is completed.

It is well known (and routine to prove) that the mappings $I: \mathscr{V} \ni K \rightarrow \sigma_{K}$ and $I^{*}: \mathscr{V}^{*} \ni K^{*} \rightarrow \sigma_{K^{*}}$, where $\sigma_{K}$ is the support function of $K: \sigma_{K}\left(x^{*}\right)=$ $\sup _{x \in K}\left\langle x, x^{*}\right\rangle$ and $\sigma_{K^{*}}(x)=\sup _{x^{*} \in K^{*}}\left\langle x, x^{*}\right\rangle$ are isometric isomorphisms respectively between $(\mathscr{V}, h)$ and $\left(F^{*}, \rho^{*}\right)$, and between $\left(\mathscr{V}^{*}, h\right)$ and $(F, \rho)$ (Minkowski's duality), where $F^{*}$ is the space of all sublinear, positively homogeneous, continuous and $w^{*}$-lower semicontinuous functionals on $E^{*}$ furnished with the uniform metric $\rho^{*}$ and $F$ is the space of all sublinear, positively-homogeneous, continuous functionals on $E$ furnished with the uniform metric $\rho$. It is easy to see that $(F, \rho)$ is a complete metric space, therefore $\left(\mathscr{V}^{*}, h\right)$ is a complete metric space too.

Let $P$ be the set of all equivalent norms in $E$, furnished with the metric $\rho$ and $P^{*}$ be the set of all equivalent dual norms in $E^{*}$ furnished with the metric $\rho^{*}$. It is a routine matter to prove that $P$ is an open subset of the complete metric space of all continuous seminorms on $E$ under the distance $\rho$ and that the map $\pi: p \mapsto p^{*}$ is a homeomorphism between $P$ and $P^{*}$, therefore $P$ and $P^{*}$ are Baire spaces. Also, the topology on $P$ (resp. on $P^{*}$ ) depends only on the topology in $E$ (resp. in $E^{*}$ ), but does not depend on the choice of the concrete equivalent norm in $E$ (resp. concrete equivalent dual norm in $E^{*}$ ).

Define the following sets:

$R=\{X \in \mathscr{V}: 0 \in \operatorname{int} X, X$ is symmetric with respect to 0$\}$, $R^{*}=\left\{X^{*} \in \mathscr{V}^{*}: 0 \in \operatorname{int} X^{*}, X^{*}\right.$ is symmetric with respect to 0$\}$.

It is easy to see that $I$ and $I^{*}$ are isometric isomorphisms respectively between $R$ and $P^{*}$ and between $R^{*}$ and $P$, when $R$ and $R^{*}$ are furnished with the Hausdorff metric.

THEOREM 4. Let E be a Banach space and let the following condition hold:

(A) there exists an equivalent norm $\|\cdot\|$ in $E$ (with dual $\|\cdot\|^{*}$ ) such that the set $L$ of denting points for $B$ is dense in $S$ and the set $L^{*}$ of $w^{*}$-denting points for $B^{*}$ is dense in $S^{*}$, where $B, B^{*}, S, S^{*}$ are respectively the closed unit balls and the unit spheres in $E$ and $E^{*}: B=\{x \in E:\|x\| \leq 1\}, B^{*}=\left\{x^{*} \in E^{*}:\left\|x^{*}\right\|^{*} \leq 1\right\}$, $S=\{x \in E:\|x\|=1\}, S^{*}=\left\{x^{*} \in E^{*}:\left\|x^{*}\right\|^{*}=1\right\}$.

Then

(a) there exist a dense $G_{\delta}$ subset $\mathscr{V}_{0} \subset \mathscr{V}$ and a dense $G_{\delta}$ subset $\mathscr{V}_{0}^{*} \subset \mathscr{V}^{*}$ such that for every $X \in \mathscr{V}_{0}$ the set of strongly exposing functionals is a dense $G_{\delta}$ subset of $E^{*}$ and for every $X^{*} \in \mathscr{V}_{0}^{*}$ the set of $w^{*}$-strongly exposing functionals is a dense $G_{\delta}$ subset of $E$.

(b) every $X \in \mathscr{V}_{0}$ is the closed convex hull of its strongly exposed points and

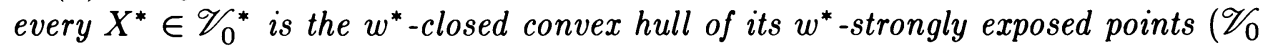
and $\mathscr{V}_{0}^{*}$ are from $\left.(\mathrm{a})\right)$

(c) there exist a dense $G_{\delta}$ subset $F_{0} \subset F$ and a dense $G_{\delta}$ subset $F_{0}^{*} \subset F^{*}$ such that every $f \in F_{0}$ is Fréchet differentiable on a dense $G_{\delta}$ subset of $E$ and every $f^{*} \in F_{0}^{*}$ is Fréchet differentiable on a dense $G_{\delta}$ subset of $E^{*}$; 
(d) there exists a dense $G_{\delta}$ subset $P_{0} \subset P$ such that: the set of dual norms $P_{0}^{*}:=\left\{p^{*} \in P^{*}: p \in P_{0}\right\}$ is a dense $G_{\delta}$ subset of $P^{*}$, every $p \in P_{0}$ is Fréchet differentiable on a dense $G_{\delta}$ subset of $E$ and every $p^{*} \in P_{0}^{*}$ is Fréchet differentiable on a dense $G_{\delta}$ subset of $E^{*}$.

ProOF. (a) By Lemma 2 there exist $L_{n} \subset L^{*}$ such that $\left\|x^{*}-y^{*}\right\|^{*} \geq 1 / n$ for every $x^{*}, y^{*} \in L_{n}, x^{*} \neq y^{*}, n=2,3, \ldots$ and $\bigcup_{n=2}^{\infty} L_{n}$ is a dense subset of $S^{*}$.

Let $n \in\{2,3, \ldots\}$ be fixed. For every $l \in L_{n}$ there exist $x_{n}(l) \in S, \alpha_{n}(l)>0$ such that $l \in S\left(B^{*}, x_{n}(l), \alpha_{n}(l)\right) \subset B(l ; 1 / 2 n)$. By Lemma 1 there exists $\varepsilon_{n}(l)>0$ such that

$$
S\left(B^{*}, x, \alpha_{n}(l) / 2\right) \subset S\left(B^{*}, x_{n}(l), \alpha_{n}(l)\right) \subset B(l ; 1 / 2 n)
$$

for every $x \in B\left(x_{n}(l) ; \varepsilon_{n}(l)\right)$. Choose a denting point $z_{n}(l) \in B\left(x_{n}(l) ; \varepsilon_{n}(l)\right) \cap L$. Then there exist $z_{n}^{*}(l) \in S^{*}, \beta_{n}(l)>0$ such that

$$
z_{n}(l) \in S\left(B, z_{n}^{*}(l), 2 \beta_{n}(l)\right) \subset B\left(x_{n}(l) ; \varepsilon_{n}(l)\right) .
$$

By Lemma 1 there exists $\gamma_{n}(l)>0$ such that

$$
S\left(B, x^{*}, \beta_{n}(l)\right) \subset S\left(B, z_{n}^{*}(l), 2 \beta_{n}(l)\right) \subset B\left(x_{n}(l) ; \varepsilon_{n}(l)\right)
$$

for every $x^{*} \in B\left(z_{n}^{*}(l) ; \gamma_{n}(l)\right)$.

Let $S_{0}^{*}$ be the set of those functionals from $S^{*}$, which attain their supremum over $S$. By the Bishop-Phelps theorem [1] $S_{0}^{*}$ is dense in $S^{*}$. Let $x_{n}^{*}(l) \in S_{0}^{*} \cap$ $B\left(z_{n}^{*}(l) ; \gamma_{n}(l)\right)$. Choose some $y \in S$ with $\left\langle y, x_{n}^{*}(l)\right\rangle=1$. By $(2) y \in B\left(x_{n}(l) ; \varepsilon_{n}(l)\right)$ and by (1) we obtain

$$
x_{n}^{*}(l) \in B(l ; 1 / 2 n)
$$

We will prove that

$$
\left\{x \in S:\left\langle x, x_{n}^{*}\left(l_{1}\right)\right\rangle=1\right\} \cap S\left(B, x_{n}^{*}\left(l_{2}\right), \beta_{n}\left(l_{2}\right)\right)=\varnothing
$$

for every $l_{1}, l_{2} \in L_{n}, l_{1} \neq l_{2}$.

Assume the contrary: for some $l_{1}, l_{2} \in L_{n}, l_{1} \neq l_{2}$ there exists

$$
\tilde{y} \in S\left(B, x_{n}^{*}\left(l_{2}\right), \beta_{n}\left(l_{2}\right)\right)
$$

for which $\left\langle\tilde{y}, x_{n}^{*}\left(l_{1}\right)\right\rangle=1$. By (3) we have $x_{n}^{*}\left(l_{1}\right) \in B\left(l_{1} ; 1 / 2 n\right)$. From $\tilde{y} \in$ $S\left(B, x_{n}^{*}\left(l_{2}\right), \beta_{n}\left(l_{2}\right)\right)$ and by (2) it follows that $\tilde{y} \in B\left(x_{n}\left(l_{2}\right) ; \varepsilon_{n}\left(l_{2}\right)\right)$. By (1) we have

$$
x_{n}^{*}\left(l_{1}\right) \in\left\{x^{*} \in B^{*}:\left\langle\tilde{y}, x^{*}\right\rangle=1\right\} \subset S\left(B^{*}, \tilde{y}, \alpha_{n}\left(l_{2}\right) / 2\right) \subset B\left(l_{2} ; l / 2 n\right),
$$

and we obtain the contradiction

$$
1 / n \leq\left\|l_{1}-l_{2}\right\|^{*} \leq\left\|l_{1}-x_{n}^{*}\left(l_{1}\right)\right\|^{*}+\left\|x_{n}^{*}\left(l_{1}\right)-l_{2}\right\|^{*}<1 / n,
$$

and (4) is proved.

Denote $H_{n m}=\left\{l \in L_{n}: \beta_{n}(l)>1 / m\right\}$ and

$\mathscr{V}_{n m k}=\left\{X \in \mathscr{V}: \exists \alpha>0, \exists \gamma>0: \operatorname{diam} S\left(X, x_{n}^{*}(l), \alpha\right)<1 / k-\gamma, \forall l \in H_{n m}\right\}$, if $H_{n m} \neq \varnothing$ and $V_{n m k}=\mathscr{V}$ if $H_{n m}=\varnothing$.

We will prove that $\mathscr{V}_{n m k}$ is a dense and open subset of $\mathscr{V}$ for every $n \in N \backslash\{1\}$, $m, k \in N$.

(1) "Denseness". Let $n \in N \backslash\{1\}, m, k \in N$ be fixed, $H_{n m} \neq \varnothing, X_{0} \in \mathscr{V}$, $\varepsilon>0, u(l) \in S\left(X_{0}, x_{n}^{*}(l), \varepsilon / 2 m\right)$ for $l \in L_{n}$. Let $v(l) \in B[u(l) ; \varepsilon]$ be such that $x_{n}^{*}(l)$ 
attains at $v(l)$ its maximum over $B[u(l) ; \varepsilon]$ (such $v(l)$ exists because of the choice of $\left.x_{n}^{*}(l)\right)$. By (4) for every $l_{1}, l_{2} \in H_{n m}, l_{1} \neq l_{2}$ we have

$$
\begin{aligned}
& \left(v\left(l_{1}\right)-u\left(l_{1}\right)\right) / \varepsilon \notin S\left(B, x_{n}^{*}\left(l_{2}\right), \beta_{n}\left(l_{2}\right)\right), \\
& \left(v\left(l_{1}\right)-u\left(l_{1}\right)\right) / \varepsilon \notin S\left(B, x_{n}^{*}\left(l_{2}\right), 1 / m\right), \\
& \left\langle v\left(l_{1}\right)-u\left(l_{1}\right), x_{n}^{*}\left(l_{2}\right)\right\rangle<\varepsilon(1-1 / m)
\end{aligned}
$$

and we obtain

$$
\begin{aligned}
& \left\langle v\left(l_{2}\right), x_{n}^{*}\left(l_{2}\right)\right\rangle-\left\langle v\left(l_{1}\right), x_{n}^{*}\left(l_{2}\right)\right\rangle=\left\langle v\left(l_{2}\right)-u\left(l_{2}\right), x_{n}^{*}\left(l_{2}\right)\right\rangle \\
& \quad+\left\langle u\left(l_{2}\right), x_{n}^{*}\left(l_{2}\right)\right\rangle-\left\langle v\left(l_{1}\right)-u\left(l_{1}\right), x_{n}^{*}\left(l_{2}\right)\right\rangle-\left\langle u\left(l_{1}\right), x_{n}^{*}\left(l_{2}\right)\right\rangle \\
& \geq \varepsilon+\sup _{z \in X_{0}}\left\langle z, x_{n}^{*}\left(l_{2}\right)\right\rangle-\varepsilon / 2 m-\varepsilon(1-1 / m)-\left\langle u\left(l_{1}\right), x_{n}^{*}\left(l_{2}\right)\right\rangle \\
& \quad \geq \varepsilon / 2 m
\end{aligned}
$$

Hence

$$
\left\langle v\left(l_{1}\right), x_{n}^{*}\left(l_{2}\right)\right\rangle \leq\left\langle v\left(l_{2}\right), x_{n}^{*}\left(l_{2}\right)\right\rangle-\varepsilon / 2 m
$$

for every $l_{1}, l_{2} \in H_{n m}, l_{1} \neq l_{2}$. Also, if $x \in X_{0}$ and $l \in H_{n m}$, then we have

$$
\begin{aligned}
\left\langle v(l), x_{n}^{*}(l)\right\rangle-\left\langle x, x_{n}^{*}(l)\right\rangle & \geq\left\langle v(l)-u(l), x_{n}^{*}(l)\right\rangle+\left\langle u(l), x_{n}^{*}(l)\right\rangle-\sup _{z \in X_{0}}\left\langle z, x_{n}^{*}(l)\right\rangle \\
& \geq \varepsilon-\varepsilon /(2 m) \geq \varepsilon /(2 m),
\end{aligned}
$$

whence

(6)

$$
\left\langle x, x_{n}^{*}(l)\right\rangle \leq\left\langle v(l), x_{n}^{*}(l)\right\rangle-\varepsilon / 2 m
$$

for every $x \in X_{0}$ and $l \in H_{n m}$.

Put $X_{1}=\operatorname{co}\left\{\left\{v(l): l \in L_{n}\right\} \cup X_{0}\right\}, X_{2}=\overline{X_{1}}$ and for $l \in H_{n m} Y(l)=$ $\left\{v\left(x^{*}\right): x^{*} \in H_{n m} \backslash\{l\}\right\} \cup X_{0}$. For $l \in H_{n m}$ and $y \in \operatorname{co} Y(l)$ we have $y=\sum_{i=1}^{r} t_{i} y_{i}$, where $t_{i} \in[0,1], 1 \leq i \leq r, \sum_{i=1}^{r} t_{i}=1, y_{i} \in Y(l)$ and by (5) and (6) we obtain

$$
\left\langle y, x_{n}^{*}(l)\right\rangle=\sum_{i=1}^{r} t_{i}\left\langle y_{i}, x_{n}^{*}(l)\right\rangle \leq\left\langle v(l), x_{n}^{*}(l)\right\rangle-\frac{\varepsilon}{2 m} .
$$

Hence

$$
\left\langle y, x_{n}^{*}(l)\right\rangle \leq\left\langle v(l), x_{n}^{*}(l)\right\rangle-\frac{\varepsilon}{2 m}
$$

for every $y \in \overline{\operatorname{co}} Y(l)$.

Since for every $l \in H_{n m}$ we have $X_{2}=\operatorname{co}\{\overline{c o} Y(l) \cup v(l)\}$, by (7), we obtain

$$
\left\langle v(l), x_{n}^{*}(l)\right\rangle=\sup _{z \in X_{2}}\left\langle z, x_{n}^{*}(l)\right\rangle, \quad \text { for every } l \in H_{n m} .
$$

Let $0<\alpha<\varepsilon / 8 m k\left(\operatorname{diam} X_{0}+2 \varepsilon\right)$ and $x \in S\left(X_{2}, x_{n}^{*}(l), \alpha\right), x \neq v(l)$. Then we can write $x=\lambda v(l)+(1-\lambda) a$ for some $\lambda \in[0,1)$ and $a \in \overline{c o} Y(l)$ and by (7), (8) we obtain

$$
\begin{aligned}
\|x-v(l)\| & =(1-\lambda)\|v(l)-a\|=\left|\left\langle v(l)-x, x_{n}^{*}(l)\right\rangle\right|\|v(l)-a\| /\left|\left\langle v(l)-a, x_{n}^{*}(l)\right\rangle\right| \\
& \leq 2 m\left(\operatorname{diam} X_{0}+2 \varepsilon\right) \alpha / \varepsilon<1 / 4 k .
\end{aligned}
$$

Therefore $\operatorname{diam} S\left(X_{2}, x_{n}^{*}(l), \alpha\right) \leq 1 / 2 k<1 / k-1 / 3 k$ for every $l \in H_{n m}$ which shows that $X_{2} \in \mathscr{V}_{n m k}$. This is the main step of the construction. 
Let $x \in X_{1}$. Then $x=\sum_{i=1}^{s} \lambda_{i} x_{i}$ for some $x_{i} \in\left\{v(1): l \in H_{n m}\right\} \cup X_{0}, \lambda_{i} \in[0,1]$, $1 \leq i \leq s, \sum_{i=l}^{s} \lambda_{i}=1$ and since the function $d\left(\cdot, X_{0}\right)$ (here $d$ and $h$ below are defined by $\|\cdot\|$ ) is convex (because $X_{0}$ is convex), we have

$$
d\left(x, X_{0}\right)=d\left(\sum_{i=1}^{s} \lambda_{i} x_{i}, X_{0}\right) \leq \sum_{i=1}^{s} \lambda_{i} d\left(x_{i}, X_{0}\right) \leq \sum_{i=1}^{s} \lambda_{i} \varepsilon=\varepsilon
$$

Since $d\left(\cdot, X_{0}\right)$ is continuous, $d\left(x, X_{0}\right) \leq \varepsilon$ for every $x \in X_{2}$, whence $h\left(X_{0}, X_{2}\right) \leq$ $\varepsilon$ and the denseness is proved.

(2) "OPENNESS". Let $X_{0} \in \mathscr{V}_{n m k}$. Then there exist $\alpha_{0}>0$ and $\gamma>0$ such that $\operatorname{diam} S\left(X_{0}, x_{n}^{*}(l), \alpha_{0}\right)<1 / k-\gamma$ for every $l \in H_{n m}$. There exists $\delta>0$ such that $\operatorname{diam} S\left(X_{0}, x_{n}^{*}(l), \alpha_{0}\right)+2 \delta<1 / k-\gamma / 2$ for every $l \in H_{n m}$ and $\alpha:=\alpha_{0}-2 \delta>0$. For every $X \in \mathscr{V}$ for which $h\left(X, X_{0}\right)<\delta$ we will show that

$$
S\left(X, x_{n}^{*}(l), \alpha\right) \subset S\left(X_{0}, x_{n}^{*}(l), \alpha_{0}\right)+\delta B,
$$

for every $l \in H_{n m}$.

Let $l \in H_{n m}$ and $x \in S\left(X, x_{n}^{*}(l), \alpha\right)$. Since $X \subset X_{0}+\delta B$ and $X_{0} \subset X+\delta B$, there exist $x_{0} \in X$ and $u \in \delta B$ such that $x=x_{0}+u$ and the following inequalities are fulfilled:

$$
\begin{aligned}
\left\langle x_{0}, x_{n}^{*}(l)\right\rangle & =\left\langle x, x_{n}^{*}(l)\right\rangle-\left\langle u, x_{n}^{*}(l)\right\rangle \geq \sup _{z \in X}\left\langle z, x_{n}^{*}(l)\right\rangle-\alpha-\delta\left\|x_{n}^{*}(l)\right\| \\
& =\sup _{z \in X}\left\langle z, x_{n}^{*}(l)\right\rangle+\sup _{z \in \delta B}\left\langle z, x_{n}^{*}(l)\right\rangle-\alpha-2 \delta \\
& =\sup _{z \in(X+\delta B)}\left\langle z, x_{n}^{*}(l)\right\rangle-\alpha_{0} \geq \sup _{z \in X_{0}}\left\langle z, x_{n}^{*}(l)\right\rangle-\alpha_{0} .
\end{aligned}
$$

Hence $x_{0} \in S\left(X_{0}, x_{n}^{*}(l), \alpha_{0}\right)$ and (9) is proved. By (9) it follows that

$$
\operatorname{diam} S\left(X, x_{n}^{*}(l), \alpha\right)<1 / k-\gamma / 2
$$

for every $l \in H_{n m}$, therefore $X \in \mathscr{V}_{n m k}$ and $\mathscr{V}_{n m k}$ is open.

It is easy to see that $\mathscr{V}_{0}:=\bigcap_{n, m, k=1}^{\infty} \mathscr{V}_{n m k}=\left\{X \in \mathscr{V}:\right.$ every $x^{*} \in M$ is strongly exposing for $X\}$, where $M=\bigcup_{n, m=1}^{\infty}\left\{x_{n}^{*}(l): l \in H_{n, m}\right\}$, and by the Baire category theorem $\mathscr{V}_{0}$ is dense $G_{\delta}$ in $\mathscr{V}$. Since $L_{n}=\bigcup_{m=1}^{\infty} H_{n m}$ and $\bigcup_{n=1}^{\infty} L_{n}$ is dense in $S^{*}$, by (3) it follows that $M$ is dense in $S^{*}$. Obviously the set $\bigcup\{t M: t>0\}$ is dense in $E^{*}$. If $x^{*}$ is a strongly exposing functional for some $X \subset E$, then obviously $t x^{*}$, $t>0$ is also a strongly exposing functional for $X$ and by Lemma 3 the first part of the assertion (a) is proved.

The proof of the second part of (a) is analogous, as the roles of $E$ and $E^{*}$ are exchanged and we need not the theorem of Bishop-Phelps. The peculiarity here is that we define $X_{2}^{\prime}={\overline{X_{1}^{\prime}}}^{*}$, where ${\overline{X_{1}^{\prime}}}^{*}$ denote the $w^{*}$-closed hull of $X_{1}^{\prime}$ and we must prove that $h\left(X_{0}^{\prime}, X_{2}^{\prime}\right) \leq \varepsilon\left(X_{0}^{\prime}\right.$ and $X_{1}^{\prime}$ are defined in $E^{*}$ in an analogous way, as the set $X_{0}$ and $\left.X_{1}\right)$.

By the construction $X_{1}^{\prime} \subset X_{0}^{\prime}+\varepsilon B^{*}$ and since the set $X_{0}^{\prime}+\varepsilon B^{*}$ is $w^{*}$-compact, we have $X_{2}^{\prime}:={\overline{X_{1}^{\prime}}}^{*} \subset X_{0}^{\prime}+\varepsilon B^{*}$, whence $h\left(X_{2}^{\prime}, X_{0}^{\prime}\right) \leq \varepsilon$.

(b) This is an immediate consequence from (a) and from the separation theorem.

(c) The assertion follows from (a) and from the well known duality between Fréchet differentiability and stror.g exposition:

$x \in X \in \mathscr{V}$ is a strongly exposed point for $X$ by $x^{*} \in E^{*}$ if and only if $\sigma_{X}$ is Fréchet differentiable at $x^{*}$, 
$x^{*} \in X^{*} \in \mathscr{V}^{*}$ is a $w^{*}$-strongly exposed point of $X^{*}$ by $x \in E$ if and only if $\sigma_{X^{*}}$ is Fréchet differentiable at $x$ (see for instance [2, p. 159]).

(d) The set $L_{n}$ from (a) can be defined (also by Zorn's lemma) in such a way, that the following additional property is fulfilled: if $l \in L_{n}$, then $-l \in L_{n}$. Now we replace $\mathscr{V}$ by $R$ and work as in the proof of (a), choosing every $y(\cdot)$ from the set $\left\{x_{n}(\cdot), z_{n}(\cdot), z_{n}^{*}(\cdot), x_{n}^{*}(\cdot), u(\cdot), v(\cdot)\right\}$ and every $\lambda(\cdot)$ from the set $\left\{\alpha_{n}(\cdot), \beta_{n}(\cdot), \gamma_{n}(\cdot)\right\}$ in such a way, that $y(-l)=-y(l)$ and $\lambda(-l)=\lambda(l)$ for every $l \in L_{n}$. Having in mind that $I$ is an isometric isomorphism between $R$ and $P^{*}$, we apply the above mentioned duality between Fréchet differentiability and strong exposition. Thus we obtain a dense $G_{\delta}$ subset $P_{1}^{*}$ of $P^{*}$ such that every $p^{*} \in P_{1}^{*}$ is Fréchet differentiable on a dense $G_{\delta}$ subset of $E^{*}$. Analogously we obtain a dense $G_{\delta}$ subset $P_{0}$ of $P$ such that every $p \in P_{0}$ is Fréchet differentiable on a dense $G_{\delta}$ subset of $E$. Put $P_{0}^{*}=P_{1}^{*} \cap \pi\left(P_{0}\right)$ and since $\pi$ is a homeomorphism, the proof is completed.

In an analogy with the definitions of Asplund and weak* Asplund spaces (see for instance [2]), we give the following definition.

DEFinition 5. A Banach space $E$ (resp. the dual $E^{*}$ of a Banach space $E$ ) will be called an almost Asplund (resp. almost weak* Asplund) space, if there exists a dense $G_{\delta}$ subset $F_{0}$ of $F$ (resp. $F_{0}^{*}$ of $F^{*}$ ) such that every $f \in F$ (resp. every $f^{*} \in F^{*}$ ) is Fréchet differentiable on a dense $G_{\delta}$ subset of $E$ (resp. of $E^{*}$ ).

Thus, in this terminology, Theorem 4 states that if for a Banach space $E$ condition (A) of Theorem 4 holds, then $E$ is an almost Asplund space and $E^{*}$ is an almost weak* Asplund space.

From the condition (d) of Theorem 4 and the results of Godefroy [8] we have the following

COROLLARY 6. If a Banach space satisfies the assumptions of Theorem 4, then there exists a dense $G_{\delta}$ subset $P_{0}$ of $P$ such that for every norm $p \in P_{0}$, when $E$ is furnished with $p$, one has

(1) there exists a unique projection of norm 1 from $E^{* * *}$ to $E^{*}$, and thus $E$ is unique isometric predual of $E^{*}$,

(2) $E$ is not isometric to a dual space (if $E$ is not reflexive).

A norm $\|\cdot\|$ of a Banach space $E$ is said to be locally uniformly rotund $(L U R)$ if for every sequence $\left\{x_{n}\right\}_{n \geq 0} \subset E$ with $\left\|x_{n}\right\| \leq 1, n=0,1,2, \ldots, \lim \left\|x_{n}+x_{0}\right\|=2$ it follows that $x_{n} \rightarrow x_{0}$.

It is not difficult to see that if the norm $\|\cdot\|$ of $E$ is $L U R$, then every point of its unit sphere is strongly exposed for its unit ball.

A result of G. Godefroy, S. Troyanski, J. Whitfield and V. Zizler [9] asserts that if $E^{*}$ is weakly compactly generated Banach space (WCG, that is there exists in $E^{*}$ a weak compact set whose linear hull is dense in $\left.E^{*}\right)$, then there exists an equivalent $L U R$ norm in $E$ whose dual norm is also $L U R$. Thus by Theorem 4(c) we obtain the following.

PROPOSITION 7. If $E^{*}$ is $W C G$, then $E^{*}$ is an almost weak ${ }^{*}$ Asplund space.

For a comparison it is worth to mention the following well-known facts: if $E^{*}$ is WCG, then $E$ is an Asplund space; also if $E$ is reflexive, then $E^{*}$ is a weak* Asplund space. 
As a corollary from Proposition 7 we obtain that the odd conjugate of the James Tree space JT, which are WCG (see for instance $[4$, p. 214]) are almost weak* Asplund spaces.

The particular case of Proposition 7, when $E^{*}$ is separable, follows from [5].

EXAMPLE 8. There exist almost weak $^{*}$ Asplund spaces which are not weak* Asplund spaces: $c_{0}$ has not the Radon-Nikodym property, therefore $l_{1}=c_{0}^{*}$ is not a weak* Asplund space. But $l_{1}$ is separable and by [5] (also by Proposition 7) $l_{1}$ is an almost weak ${ }^{*}$ Asplund space. There are almost Asplund spaces which are not Asplund: for example every separable Banach space which dual is not separable (for instance $l_{1}$ ) is not an Asplund space, but it is an almost Asplund space (this follows from the results in [5] and from the duality between Fréchet differentiability and strong exposition).

QUESTION 9 What are the necessary and sufficient conditions for $E\left(E^{*}\right)$ to be an almost Asplund (almost weak* Asplund) space?

ACKNOWLEDGEMENTS The author would like to thank Professor P. S. Kenderov for his helpful suggestions and the encouragement while this work was in progress. The author is very grateful to the referee for observing the validity of Corollary 6 .

\section{REFERENCES}

1. E. Bishop and R. R. Phelps, The support functionals of a convex set, Convexity, Proc. Sympos. Pure Math., vol. 7, Amer. Math. Soc., Providence, R. I., 1963, pp. 27-35.

2. R. D. Bourgin, Geometric aspects of convex sets with the Radon-Nikodym property, Lecture Notes in Math., vol. 993, Amer. Math. Soc., Providence, R. I., 1983.

3. J. Diestel, Geometry of Banach spaces-selected topics, Lecture Notes in Math., vol. 485, Springer-Verlag, Berlin and New York, 1975.

4. J. Diestel and J. Uhl, Vector measures, Math. Surveys, vol. 15, Amer. Math. Soc., Providence, R. I., 1977.

5. P. Gr. Georgiev, Almost all closed, convex and bounded subsets of a Banach space are dentable, Proc. 14th Spring Conf. Union Bulg. Math., 1985, 355-361.

6. J. R. Giles, Convex analysis with application in differentiation of convex functions, Pitman Advanced Publ. Prog., 1982.

7. J. R. Giles, P. A. Gregory and B. Sims, Characterization of normed linear spaces with Mazur's intersection property, Bull. Austral. Math. Soc. 18 (1978), 105-123.

8. G. Godefroy, Points de Namioka, espaces normants, applications a la theorie isometrique de la dualite, Israel J. Math. 38 (1981), 209-220.

9. G. Godefroy, S. Troyanski, J. Whitfield and V. Zizler, Locally uniformly rotund renorming and injections into $c_{0}(\Gamma)$, Canad. Math. Bull. 27 (1984), 494-500.

10. K. Kuratowski, Topology I, "Mir", Moscow, 1966. (Russian)

11. S. Mazur, Über schwach Konvengenz in den Raumen $\left(L^{p}\right)$, Studia Math. 4 (1933), 128-133.

12. R. R. Phelps, A representation of bounded convex sets, Proc. Amer. Math. Soc. 11 (1960), 976-983.

13. F. Sullivan, Dentability, smoothability and stronger properties in Banach spaces, Indiana Math. J. 26 (1977), 545-553.

Bulgarian ACademy of Sciences, institute of Mathematics, 1090 Sofia, P. O. BOX 373, BULGARIA 